\title{
A SIMPLIFIED FORM FOR NEARLY REDUCIBLE AND NEARLY DECOMPOSABLE MATRICES
}

\section{J. HARTFIEL}

Introduction and definitions. Nearly reducible and nearly decomposable matrices have been discussed in [4], [5], and [6]. Results in these papers were obtained by using a canonical form for these matrices. In this paper we give a simplification of this canonical form. It is then shown that the main result in [5], a major result in [4] and a result in [2] as well as other properties of nearly reducible and nearly decomposable matrices are more easily obtained.

In the paper the following definitions concerning matrix theory are used.

An $n \times n$ matrix $A$ is said to be reducible if there exists a permutation matrix $P$ so that

$$
P^{t} A P=\left(\begin{array}{ll}
B & O \\
C & D
\end{array}\right)
$$

where $B$ and $D$ are square. Otherwise $A$ is said to be irreducible. $E_{i j}$ is the $n \times n$ matrix which has a one in the $(i, j)$ position and 0 's elsewhere. If $A$ is irreducible and for each $a_{i j} \neq 0, A-a_{i j} E_{i j}$ is reducible, then $A$ is said to be nearly reducible. An $n \times n$ matrix $A$ is said to be partly decomposable if there exist permutation matrices $P$ and $Q$ so that

$$
P A Q=\left(\begin{array}{ll}
B & O \\
C & D
\end{array}\right)
$$

where $B$ and $D$ are square. If no such $P$ and $Q$ exist, the matrix is said to be fully indecomposable. If $A$ is fully indecomposable and for each $a_{i j} \neq 0, A-a_{i j} E_{i j}$ is partly decomposable, then $A$ is said to be nearly decomposable. $A$ is said to have a positive diagonal if there exist positive entries $a_{1 \sigma(1)}, a_{2 \sigma(2)}, \cdots, a_{n \sigma(n)}$ where $\sigma$ is a permutation of $1,2, \cdots, n$. An entry $a_{i j}$ is said to lie on this positive diagonal if $\sigma(i)=j$. If $a_{i_{1} j_{1}}, a_{i_{1} j_{2}}, a_{i_{2} j_{2}}, \cdots, a_{i_{m} i_{m}}=a_{i_{1} j_{1}}$ are distinct nonzero elements in $A$, then $A$ is said to have a loop.

The following definitions concerning graph theory are used in the paper.

A graph $G$ is said to be strongly connected if for each pair of distinct vertices $x$ and $y$, there is a path from $x$ to $y$. If a graph is

Received by the editors March 14, 1969. 
strongly connected and with the removal of any arc it loses this property, the graph is called minimally connected. An $n \times n(0,1)$ matrix $A=\left(a_{i j}\right)$ is said to be associated with a graph $G$ having vertices $x_{1}, x_{2}, x_{3}, \cdots, x_{n}$ if and only if for each $a_{i j}=1$, we have an arc from the vertex $x_{i}$ to the vertex $x_{j}$. If $G$ is a strongly connected graph then each vertex lies on an elementary circuit. If an elementary circuit contains all the vertices of the graph then it is called a full elementary circuit. If $A$ is a subgraph of the graph $G$, then we shrink $A$ by deleting the arcs connecting any two vertices of $A$, and by identifying all vertices of $A$ with a single one of the vertices. It is easily shown that if $G$ is a minimally connected graph, then each elementary circuit is a subgraph of $G$, hence for these graphs we may shrink elementary circuits.

The importance of the paper is in the following theorem concerning the form of nearly reducible and nearly decomposable matrices. The proof will be given later.

Theorem *. Suppose $A$ is an $n \times n$ nearly decomposable [nearly reducible] $(0,1)$-matrix with $n>1$. Then there exist permutation matrices $P$ and $Q[a$ permutation matrix $P]$ and an integer $s>1$ so that

$$
\begin{gathered}
P A Q \\
\left(P^{t} A P\right)
\end{gathered}=\left(\begin{array}{ccccc}
A_{1} & 0 & \cdots & 0 & E_{1} \\
E_{2} & A_{2} & \cdots & 0 & 0 \\
\cdots & \cdots & \cdots & \cdots & \cdots \\
0 & 0 & \cdots & A_{s-1} & 0 \\
0 & 0 & \cdots & E_{s} & A_{s}
\end{array}\right)
$$

where each $E_{i}$ has exactly one entry equal to one and each $A_{i}$ is nearly decomposable [nearly reducible]. Further $A_{1}, A_{2}, \cdots, A_{s-1}$ are all $1 \times 1$.

This is a simplified form for nearly decomposable [nearly reducible] matrices in the sense that the previous form for nearly decomposable [nearly reducible] matrices (see [4], [5]) did not assert $A_{1}, A_{2}, \cdots, A_{s-1}$ to be $1 \times 1$.

Results and consequences. We exhibit two theorems concerning nearly decomposable matrices. The first is a slight improvement of that of Sinkhorn in [4]. For this we include the following lemma.

Lemma. If $A$ is a fully indecomposable $(0,1)$-matrix, then each positive entry lies on a positive diagonal. If any 0 entry is replaced by 1 then this 1 lies on a positive diagonal. (See [4, p. 199].)

Theorem 1. If $A$ is a nearly decomposable $(0,1)$-matrix and if $A$ 
has $m$ rows with three ones in it and $n-m$ rows with two ones in it then per $A \geqq m+2$. [In [4] Sinkhorn proved per $A \geqq m$.]

Proof. The proof is by induction on the dimension of $A$. If $A$ is $2 \times 2$ it is obvious since $A$ must be

$$
\left(\begin{array}{ll}
1 & 1 \\
1 & 1
\end{array}\right)
$$

Suppose the theorem holds for all nearly decomposable matrices of dimension $k$ where $2 \leqq k \leqq n-1$. Now suppose $A$ is an $n \times n$ nearly decomposable $(0,1)$-matrix. We may assume it has the following form

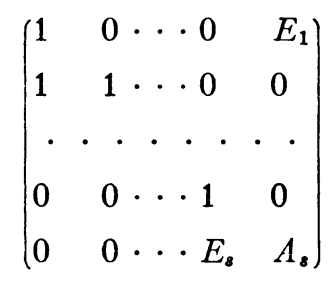

where $s>1$. If $A_{s}$ is one by one, the theorem holds. If $A_{s}$ is not $1 \times 1$ then since $A_{s}$ satisfies the induction hypothesis per $A_{s} \geqq(m-1)+2$ $=m+1$. Since the one in $E_{1}$ lies on a positive diagonal we have per $A \geqq m+2$.

Let $\Lambda_{n}$ denote the set of all $n \times n$ matrices having exactly three ones in each row and column. Sinkhorn [4] has shown that if $A \in \Lambda_{n}$ then per $A \geqq n$ and that the minimum occurs on a fully indecomposable matrix. In [2] Brualdi and Newman have shown that if $A \in \Lambda_{n}$ is a circulant matrix then per $A \geqq n+3$. We show in fact that this is always the case.

Theorem 2. If $A \in \Lambda_{n}$ then per $A \geqq n+3$.

Proof. By the above remarks we need only prove the theorem when $A$ is fully indecomposable. If a certain set of 1 's in $A, k$ in number, are replaced by 0's there results a nearly decomposable matrix $A^{\prime}$ having at least two 1 's in each row and column. We list two cases:

Case I. $k=2$. In this case we may suppose

$$
A^{\prime}=\left(\begin{array}{ll}
1 & E_{1} \\
E_{2} & A_{2}
\end{array}\right)
$$

By Theorem 1 we have per $A^{\prime} \geqq n$. By replacing two 0 's in $A^{\prime}$ by $1^{\prime}$ s to yield $A$ we see by the lemma that each of these 1's is individually on a positive diagonal and that they share a positive diagonal, hence per $A \geqq n+3$. 
Case II. $k>2$. Since $A^{\prime}$ is fully indecomposable we may assume it has a positive main diagonal. Hence we may view $A=I+P_{1}+P_{2}$ where $I$ is the identity matrix and $P_{1}, P_{2}$ permutation matrices. From this we see that when the $k 1$ 's were replaced by 0 's, at least two of these were from $P_{1}$ or $P_{2}$. Now by Theorem 1 per $A^{\prime} \geqq(n-k)+2$. By replacing the $k 0$ 's in $A^{\prime}$ by $1^{\prime}$ 's to yield $A$ we see from the lemma and the fact that at least two of these 1's share a diagonal, that

$$
\text { per } A \geqq[(n-k)+2]+k+1=n+3 \text {. }
$$

We now exhibit two theorems concerning nearly reducible matrices. Theorem 3 is not found in the literature. We include it to show that the proof using the simplified form is simpler than that needed if this form were not known.

Theorem 3. If $A$ is a nearly reducible $(0,1)$-matrix, then $A$ does not have any loops.

Proof. The proof is by induction on the dimension $n$ of $A$. By inspection, the theorem holds for $n \in\{1,2\}$. Suppose the theorem is true for all nearly reducible $(0,1)$-matrices $A$ of dimension $k ; 1 \leqq k$ $\leqq n-1$. Now let $A$ be a nearly reducible $(0,1)$-matrix of dimension $n$. We may assume $A$ has the form

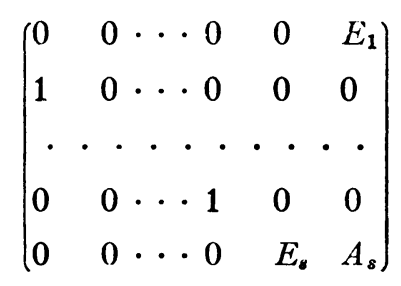

where $s>1$. Since by the induction hypothesis $A_{s}$ has no loops we see that the theorem follows.

Finally we prove a theorem which is the major result in [5].

Theorem 4. If $A$ is nearly reducible and doubly stochastic then $A$ is a full cycle permutation matrix.

Proof. We may suppose that $A$ has the form

$$
\left(\begin{array}{ccccc}
0 & 0 & \cdots & 0 & E_{1} \\
1 & 0 & \cdots & 0 & 0 \\
\cdots & \cdot & \cdots & \cdot & \cdot \\
0 & 0 & \cdots & 0 & 0 \\
0 & 0 & \cdots & E_{*} & A_{s}
\end{array}\right)
$$


where $s>1$. Since $E_{1}$ has a one in it we see that $A_{s}$ has a column of 0 's. Hence $A_{s}=(0)$ and so $A$ is a full cycle permutation matrix.

We conclude the paper with the proof of Theorem *.

Proof. First we show that a nearly reducible matrix $A$ has the stated form. The following lemma is used.

Lemma. $A$ is irreducible if and only if the graph of $A$ is strongly connected [7, p. 20].

From the lemma we see that the graph $G$ associated with $A$ is minimally connected. We now include a lemma concerning minimally connected graphs.

Lemma. In a minimally connected graph $G$, let $A$ be a set of vertices determining a strongly connected subgraph; the shrink of $A$ leads to a minimally connected graph $[1$, p. 123].

Select some vertex $v_{1}$ of $G$. If it is on a full elementary circuit then we are through. If not take some elementary circuit which contains $v_{1}$ and shrink it. Call this graph $G_{1}$. Suppose that the elementary circuit shrank to $v_{1}^{\prime}$ of $G_{1}$, If $v_{1}^{\prime}$ is not on a full elementary circuit of $G_{1}$, then take some elementary circuit it is on and shrink it. Call this graph $G_{2}$. After $m$ shrinkings like this we get a graph of a full elementary circuit. Call this graph $G_{m}$. By construction we see that if $\ell$ vertices of $G$ shrank to 1 vertex of $G_{m}$, then the remaining $n-\ell$ vertices were never shrunk.

$G$

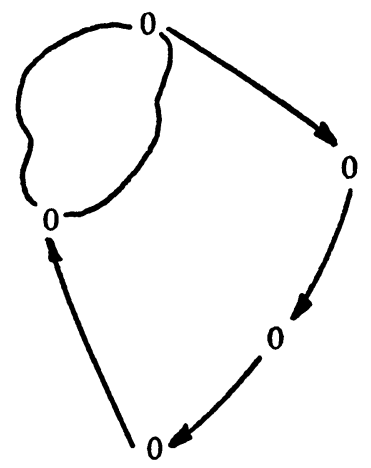

$G_{m}$

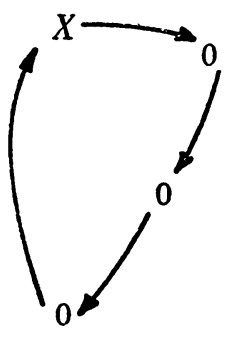

It is easily seen that the subgraph of the set of vertices which was shrunk into the one vertex is a minimally connected graph. 
From this we know that there exists a permutation matrix $P$ so that $P^{t} A P$ has the form mentioned in Theorem * for nearly reducible matrices.

We now show that a nearly decomposable matrix may be reduced to the stated form. If $A$ is nearly decomposable, take permutation matrices $P$ and $Q$ such that $P A Q=\bar{A}$ has a positive main diagonal.

Lemma. Let $A$ be an $n \times n$ matrix. Then $A$ is fully indecomposable if and only if there exists permutation matrices $P$ and $Q$ such that $P A Q$ has a positive main diagonal and is irreducible [3, p. 33].

From this lemma we see that $\bar{A}-I$ is nearly reducible. Hence there exists a permutation matrix $R$ so that $R(\bar{A}-I) R^{t}$ has the form stated in Theorem $*$ for nearly reducible matrices. Hence $R \bar{A} R^{t}$ has the form

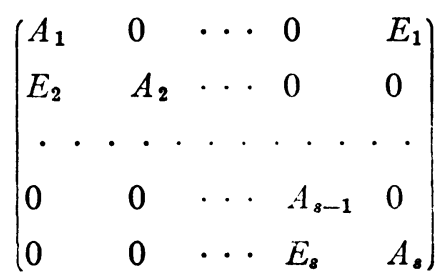

where $s>1$. Each $A_{k}$ is now fully indecomposable by the above lemma.

Lemma. Suppose $A$ is an $n \times n$ nearly decomposable $(0,1)$-matrix having the above form where each $A_{k}$ is fully indecomposable. Then each $A_{k}$ is nearly decomposable [6, p. 69].

From this lemma we see that a nearly decomposable matrix may be reduced to the form stated in Theorem $*$.

\section{REFERENCES}

1. Claude Berge, Theory of graphs, Wiley, New York, 1966.

2. R. A. Brualdi and M. Newman, Some theorems on the permanent, J. Res. Nat. Bur. Standards Sect. B 69 (1965), 159-163. MR 32 \#7427.

3. Richard Brualdi, Seymour Parter and Hans Schneider, The diagonal equivalence of a nonnegative matrix to a stochastic matrix, J. Math. Anal. Appl. 16 (1966), 31-50. MR 34 \#5844.

4. Richard Sinkhorn, Concerning a conjecture of Marshall Hall, Proc. Amer. Math. Soc. 21 (1969), 192-201.

5. Richard Sinkhorn and Mark Hedrick, Concerning nearly reducible doubly stochastic matrices (to appear).

6. Richard Sinkhorn and Paul Knopp, Problems involving diagonal products in nonnegative matrices, Trans. Amer. Math. Soc. 136 (1969). 67-75.

7. Richard Varga, Matrix iterative analysis, Prentice-Hall, Englewood Cliffs, N. J., 1962. MR $28 \# 1725$.

UNIVERSITY OF HoustoN 